\title{
Zwitterionic cocrystal of diclofenac and L-proline: Structure determination, solubility, kinetics of cocrystallization, and stability study
}

\author{
Ilma Nugrahani $^{\mathrm{a}, *}$, Dwi Utami ${ }^{\mathrm{a}, \mathrm{c}}$, Slamet Ibrahim $^{\mathrm{a}}$, Yuda Prasetya Nugraha ${ }^{\mathrm{b}}$, Hidehiro Uekusa ${ }^{\mathrm{b}}$ \\ a School of Pharmacy, Bandung Institute of Technology, Bandung, Jawa Barat 40132, Indonesia \\ ${ }^{\mathrm{b}}$ Department of Chemistry, School of Science, Tokyo Institute of Technology, Tokyo 152-8550, Japan \\ ${ }^{\mathrm{c}}$ Faculty of Pharmacy, Ahmad Dahlan University, Yogyakarta 55166, Indonesia
}

\section{A R T I C L E I N F O}

\section{Keywords:}

Diclofenac

L-Proline

Cocrystal

Solubility

Co-grinding

Stability

\begin{abstract}
A B S T R A C T
In recent decades, the design of cocrystals has developed significantly due to the unique characteristics and advantages of cocrystals, which help to improve the physicochemical properties of drugs, especially solubility. Zwitterions are attractive and interesting co-formers. However, the physicochemical properties of cocrystals with zwitterionic co-formers, i.e. zwitterionic cocrystals, have not been adequately evaluated. In this study, solid-state characterization of a newly developed zwitterionic cocrystal of diclofenac (DFA), a non-steroidal antiinflammatory drug, and the amino acid L-proline (PRO) was performed using Fourier-transform infrared spectroscopy, differential scanning calorimetry, and powder X-ray diffraction (PXRD) analyses. In addition, the crystal structure of the cocrystal (DFA-PRO) was determined by single-crystal X-ray diffraction analysis, after which the zwitterionic structure was confirmed. The cocrystallization during co-grinding, which was investigated by PXRD, followed first-order kinetics. Furthermore, the solubility of the zwitterionic cocrystals was 7.5-times higher than that of the DFA crystals. The results indicate that the cocrystal is stable under ambient conditions; however, it hydrates and transforms into a mixture of L-proline monohydrate crystals and DFA crystals under conditions of high humidity.
\end{abstract}

\section{Introduction}

Cocrystal engineering is a method for developing the most desirable physicochemical properties of drugs. It is used to optimize drug solubility, dissolution, and absorption (Bhandaru et al., 2015; He et al., 2016). Most cocrystals of medicinal compounds that have been studied are either molecular or ionic (Duggirala et al., 2016). Zwitterionic cocrystals are a special kind of cocrystals that are formed by hydrogen bond interactions between a drug and a zwitterion co-former molecule. Zwitterion co-formers allow for the formation of charge-assisted hydrogen bonds (Tilborg et al., 2014); therefore, zwitterionic cocrystal designs provide promising results in the production of raw drug materials with better physicochemical properties.

The aim of this study was to design a pharmaceutical zwitterionic cocrystal of the non-steroidal anti-inflammatory drug diclofenac in order to improve its physicochemical properties, particularly solubility. Diclofenac is widely used in both solid and liquid formulations (Barbato et al., 2003). However, its effectiveness is limited by its low aqueous solubility, which reduces its bioavailability in the body.

The zwitterion co-former selected for use in this study was L-proline
(PRO), which is a GRAS (Generally Regarded as Safe) compound and a popular amino acid used in cocrystal research (Tilborg et al., 2013; Othman et al., 2016; He et al., 2016; Liu et al., 2016). It is reported that the 5-membered ring "lateral chain" in PRO renders the molecular structure restricted and rigid (Tilborg et al., 2014). Cocrystals of the following drugs and L-proline have been formulated and studied by single-crystal X-ray diffraction (SCXRD): naproxen (Tilborg et al., 2013), flurbiprofen (Silva et al., 2016), quercetin (He et al., 2016), and nitrofurantoin (He et al., 2016). The chemical structures of diclofenac acid (DFA) and L-proline (PRO) are presented in Fig. 1.

Diclofenac ( $\mathrm{pKa}=4.18$ ) is a weak acid that is mainly available in its salt form (Fini et al., 2012). Its sodium, potassium (Williams and Buvanendran, 2011), and alkyl-hydroxyl amine (Fini et al., 2010) salts are the most common. Although the crystal structures of several DFA cocrystals, such as DFA-isonicotinic amide (Báthori et al., 2011) and DFA-theophylline (Surov et al., 2014), are known, there is no report on the crystal structure of any of the zwitterionic cocrystals. Thus, in this research, we aimed to characterize zwitterionic DFA-PRO cocrystal and confirm its structure using various methods. The crystal structure of the cocrystal was determined by SCXRD, which is used to explain the

\footnotetext{
* Corresponding author.

E-mail address: ilma_nugrahani@fa.itb.ac.id (I. Nugrahani).
} 


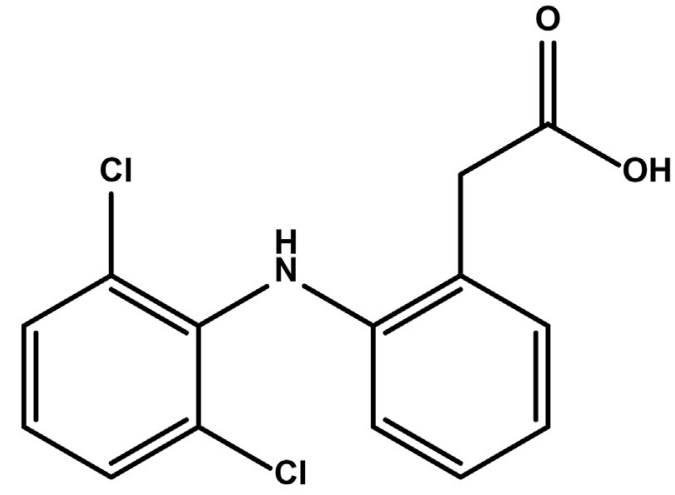

(a)

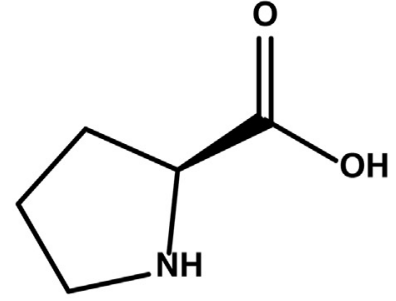

(b)

Fig. 1. Chemical structures of (a) DFA and (b) PRO.

physicochemical properties of compounds. Furthermore, the kinetics of cocrystallization during co-grinding was performed to fully study the molecular interaction between DFA and PRO. A stability study was also performed to investigate the impact of humidity on the storage of the crystal. We believe that the data obtained in this study on the production and stability of DFA-PRO will be valuable in the development of new dosage forms.

\section{Material and methods}

\subsection{Materials}

Diclofenac sodium (purity, $>99 \%$ ) was obtained from Phapros (Jakarta, Indonesia). L-Proline monohydrate (purity, > 99\%) was purchased from Xi'an ZhongYun Biotechnology Co., Ltd. (XianYang, China). Sodium chloride, potassium chloride, and potassium sulfate were purchased from Sigma-Aldrich Corporation (St. Louis, MO, USA). All solvents used were purchased from E. Merck (Darmstadt, Germany). DFA was obtained from the hydrolysis of diclofenac sodium using $1 \mathrm{M}$ hydrochloric acid.

\subsection{Methods}

\subsubsection{Cocrystal preparation}

The cocrystal was prepared by mixing DFA with PRO at a stoichiometric molar ratio of $1: 1$. The mixture was ground with a mortar and pestle and kneaded, during which five drops of ethanol were added. The kneading was continued until the powder was dry for about $30 \mathrm{~min}$. Solvent addition was continuously repeated every $10 \mathrm{~min}$. A single crystal of DFA-PRO was prepared by dissolving the kneaded product in ethanol. The resulting solution was maintained to evaporate at the ambient temperature in a sealed vial for five days, which yielded needle-shaped crystals that were suitable for SCXRD analysis.

\subsubsection{Fourier-transform infrared spectroscopy (FTIR) analysis}

The FTIR spectra of the crystals were recorded using an infrared spectrophotometer (4200 type A; JASCO, Easton, MD, USA) and a $\mathrm{KBr}$ beam splitter. The $\mathrm{KBr}$ pellet method was used to obtain background spectra. The range was set from 450 to $4000 \mathrm{~cm}^{-1}$ at $4 \mathrm{~cm}^{-1}$ resolution. The pure drug, co-former, and a physical mixture of the two compounds were also analyzed by FTIR.

\subsubsection{Differential scanning calorimetry (DSC)}

Thermal property measurements were performed using DSC 8230L (Rigaku Corporation, Tokyo, Japan) as follows. Approximately 5-10 mg of sample was accurately weighed and placed in an aluminum pan. The sample pan was heated at a rate of $3{ }^{\circ} \mathrm{C} / \mathrm{min}$ from 25 to $300{ }^{\circ} \mathrm{C}$ under a nitrogen purge of $50 \mathrm{~mL} / \mathrm{min}$. The empty aluminum pan was used as a reference. All pans were closed during the analysis.

\subsubsection{Powder $X$-ray diffraction (PXRD) analysis}

PXRD was performed using a SmartLab X-ray diffractometer (Rigaku Corporation). Each sample was placed between Mylar ${ }^{\circledast}$ films prior to analysis. Powder pattern was collected from $2 \theta=3^{\circ}$ to $40^{\circ}$ at ambient temperature at step and scan speeds of $0.01^{\circ}$ and $3^{\circ} \mathrm{min}^{-1}$, respectively, using a Cu-Ka source at $45 \mathrm{kV}$ and $200 \mathrm{~mA}$.

\subsubsection{SCXRD analysis and refinement}

SCXRD data were collected at $93 \mathrm{~K}$ for the DFA-PRO crystals. The measurement was carried out in $\omega$-scan mode with an R-axis RAPID II diffractometer (Rigaku) using the $\mathrm{Cu}-\mathrm{K} \alpha$ radiation obtained from a rotating anode source with a graphite monochromator. Integrated and scaled data were empirically corrected for absorption effects using ABSCOR (Higashi, 1995). The initial structure was solved using a dualspace algorithm implemented in SHELXT (Sheldrick, 2015a) and refined on $F^{2}$ with SHELXL-2017/1 (Sheldrick, 2015b). All non-hydrogen atoms were refined anisotropically. Hydrogen atoms attached to oxygen or nitrogen atoms were located using the differential Fourier map and refined isotropically. Other hydrogen atoms were determined geometrically and included in the calculation using the riding model. Molecular graphics were produced using Mercury 3.7 (Bruno et al., 2002; Macrae et al., 2006; Macrae et al., 2008).

\subsubsection{Powder solubility study}

An excess amount of DFA and DFA-PRO (1:1) cocrystal was produced by liquid-assisted grinding with the addition of several drops of ethanol. The powder was suspended in $10 \mathrm{~mL}$ of distilled water in capped glass vials, after which the slurries obtained were agitated in an orbital shaker ( $150 \mathrm{rpm} / \mathrm{min}$ ) at room temperature for $24 \mathrm{~h}$. Next, each suspension was filtered through a paper filter and the amount of drug solubilized was analyzed spectrophotometrically (UV-1800; Shimadzu, Kyoto, Japan) at $275 \mathrm{~nm}$. Each experiment was conducted in triplicate.

\subsubsection{Kinetics of cocrystallization during co-grinding}

Samples used in the kinetics of cocrystallization during co-grinding experiment were prepared with an automatic grinder (mortar RM 100; Retsch, Haan, Germany) at 5 scales with solvent addition. Grinding was carried out for predefined time periods of 2 to $90 \mathrm{~min}$. The samples were used in the investigation of the dynamics of interaction by PXRD analysis. 


\subsubsection{Stability study}

A simple cocrystal stability study was performed in jars that were conditioned to equilibrate at $75 \%, 80 \%$, and $90 \%$ relative humidity (RH) using saturated sodium chloride, potassium chloride, and potassium sulfate solutions, respectively. Each sample was then stored at $30{ }^{\circ} \mathrm{C}$. The powder X-ray diffractograms of the cocrystals during storage were then studied to evaluate changes in cocrystal amount in order to assess deformation. Samples were taken at 2, 4, 12, and $24 \mathrm{~h}$ after storage and assessed. Cocrystal percentage was determined using a constructed calibration curve.

Cocrystal formation was calculated using PXRD quantitative methods reported for blends of polymorphs (Qiu et al., 2015; Tiwari et al., 2007). Calibration standards were prepared by blending DFA and PRO in a 1:1 M ratio with the required amount of DFA-PRO cocrystals. The cocrystals used in the calibration were prepared by the solution method described above. The total mass of each standard was $40 \mathrm{mg}$. The standards prepared contained $0 \%, 30 \%, 50 \%, 70 \%$, or $100 \%$ (by weight) of DFA-PRO cocrystals. The standards were prepared by grinding each component individually for $2-3 \mathrm{~min}$ at room temperature in a mill. This was done to reduce particle size differences between these components and to obtain a homogeneous blend for the standards. The samples were also prepared by grinding them for 2-3 min. The standards and the samples were then analyzed using the SmartLab $\mathrm{X}$-ray diffractometer. Quantitative estimations were done using a curvefitting algorithm with a Gaussian-Lorentzian function. The proportion of the corresponding peak was stated as area under the curve. The calibration curve obtained was then validated using the parameter standards. The $\mathrm{R}^{2}$ value for each calibration curve was 0.99 . The calibration curve was used to determine the amount (\%) of cocrystal during the grinding process and storage.

\subsection{Results and discussion}

\subsubsection{Analysis of DFA-PRO cocrystals by FTIR, DSC, and PXRD}

The FTIR spectra of DFA, PRO, the physical mixture, and DFA-PRO cocrystals (1:1) are presented in Fig. 2. Analysis of the spectrum of DFA showed distinctive bands at 3324,1693 , and $937 \mathrm{~cm}^{-1}$, which respectively represent $\mathrm{O}-\mathrm{H}$ and $\mathrm{C}-\mathrm{O}$ stretching of the carboxylic acid, and $\mathrm{O}-\mathrm{H}$ vibrations out of the plane from the carboxylic acid group. The distinctive peaks of PRO were observed at 1619, 2348, and $3486 \mathrm{~cm}^{-1}$, representing $\mathrm{C}-\mathrm{O}$ stretching of the carboxylic acid, $\mathrm{N}-\mathrm{H}$ stretching of the amine heterocyclic ring, and $\mathrm{O}-\mathrm{H}$ stretching of the carboxylic acid, respectively. As seen in Fig. 2, the spectrum of the physical mixture of DFA and PRO clearly showed almost all the

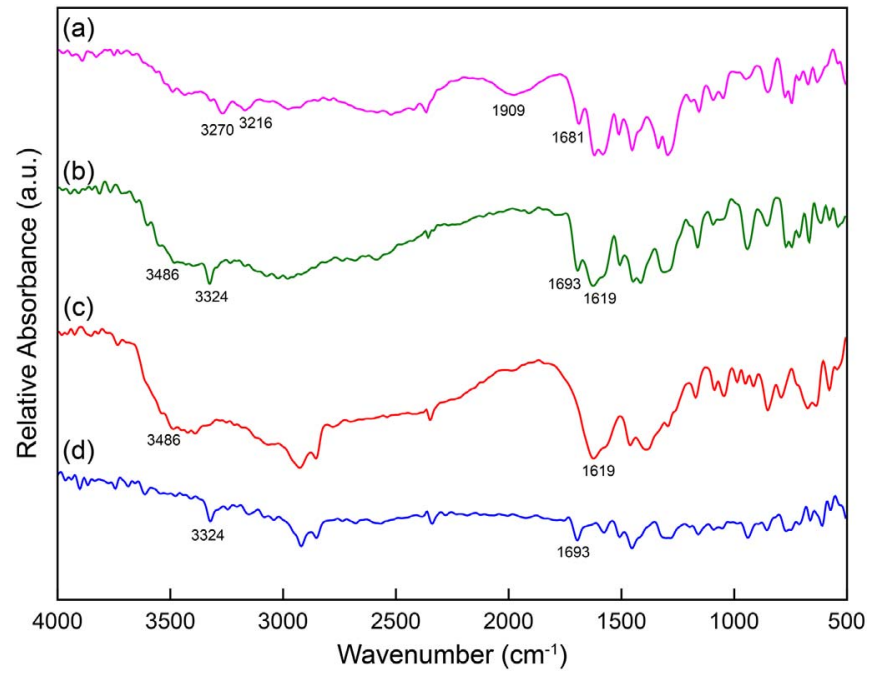

Fig. 2. FT-IR spectra of (a) DFA-PRO, (b) physical mixture of DFA and PRO (1:1), (c) PRO, and (d) DFA.

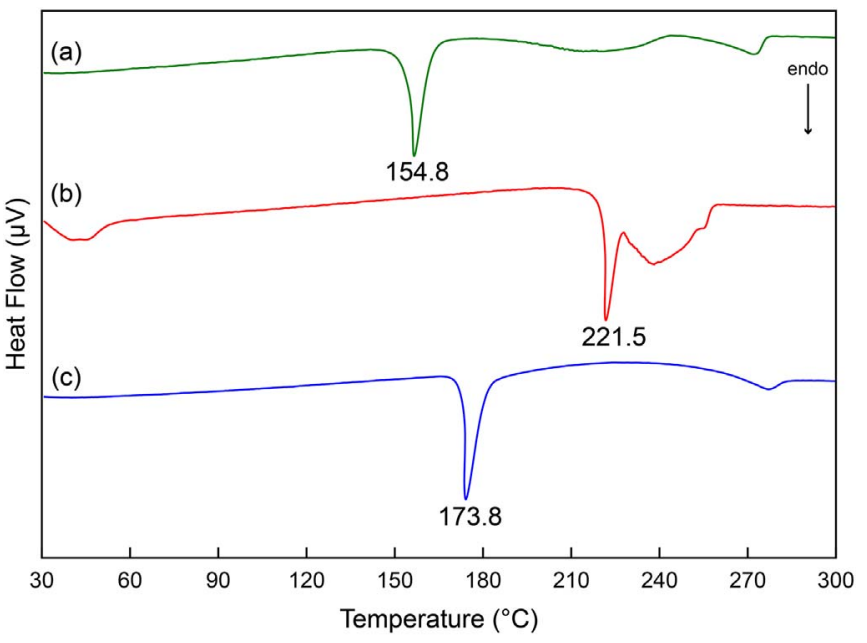

Fig. 3. DSC thermograms of (a) DFA-PRO, (b) PRO, and (c) DFA.

characteristic bands of DFA and PRO.

The first peak represents the carbonyl group of the carboxylic acid in DFA-PRO. Analysis of the spectra shows a wave number shift from $1693 \mathrm{~cm}^{-1}$ (DFA) to $1681 \mathrm{~cm}^{-1}$ (DFA-PRO) for the carbonyl group, which is indicative of hydrogen bond formation between DFA and PRO. This is supported by the shift in the wave number of the OH-free carboxylic acid in DFA from $3324 \mathrm{~cm}^{-1}$ to $3270 \mathrm{~cm}^{-1}$. The new broad spectra at $1905 \mathrm{~cm}^{-1}$ and $2541 \mathrm{~cm}^{-1}$ were generated from the hydrogen bond in O...H-N (heterocyclic) or O-H...N (heterocyclic).

The DSC thermogram of DFA, PRO, and DFA-PRO cocrystals are presented in Fig. 3. They clearly show that the melting point of DFAPRO cocrystals is lower than that of DFA $\left(173.8^{\circ} \mathrm{C}\right)$ or PRO $\left(221.5^{\circ} \mathrm{C}\right)$.

The X-ray diffractogram pattern of the DFA-PRO cocrystals was compared to those of DFA, PRO, and a mixture of both (Fig. 4). The results show that the diffraction pattern of the cocrystals is absolutely different from that of the physical mixture. There were some characteristic diffraction peaks at $2 \varphi=4.29^{\circ}, 9.73^{\circ}, 11.54^{\circ}, 13.18^{\circ}, 14.31^{\circ}$, $19.42^{\circ}, 20.48^{\circ}$, and $25.46^{\circ}$. On the other hand, characteristic diffraction peaks of DFA and PRO are $2 \theta=10.79^{\circ}, 13.31^{\circ}, 15.10^{\circ}, 18.82^{\circ}, 24.34^{\circ}$ and $8.5^{\circ}, 15.17^{\circ}, 17.98^{\circ}, 24.73^{\circ}$, respectively.

\subsubsection{SCXRD analysis}

The DFA-PRO cocrystals were needle-shaped (Fig. 5). DFA-PRO crystallized in the monoclinic chiral $P 2_{1}$ space group with one molecule

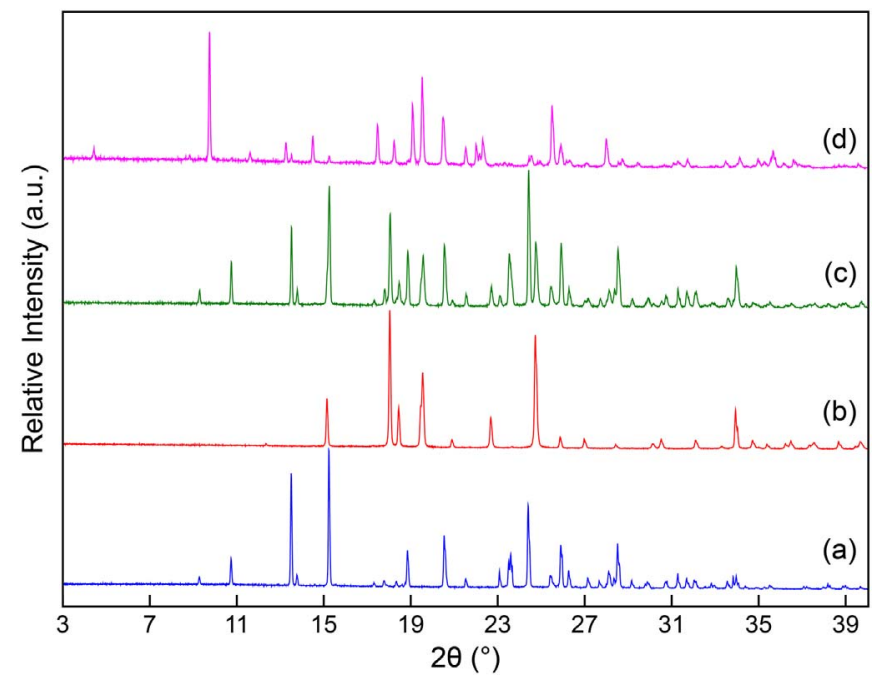

Fig. 4. PXRD patterns of (a) DFA, (b) PRO, (c) physical mixture of DFA and PRO (1:1), and (d) DFA-PRO cocrystals (1:1). 

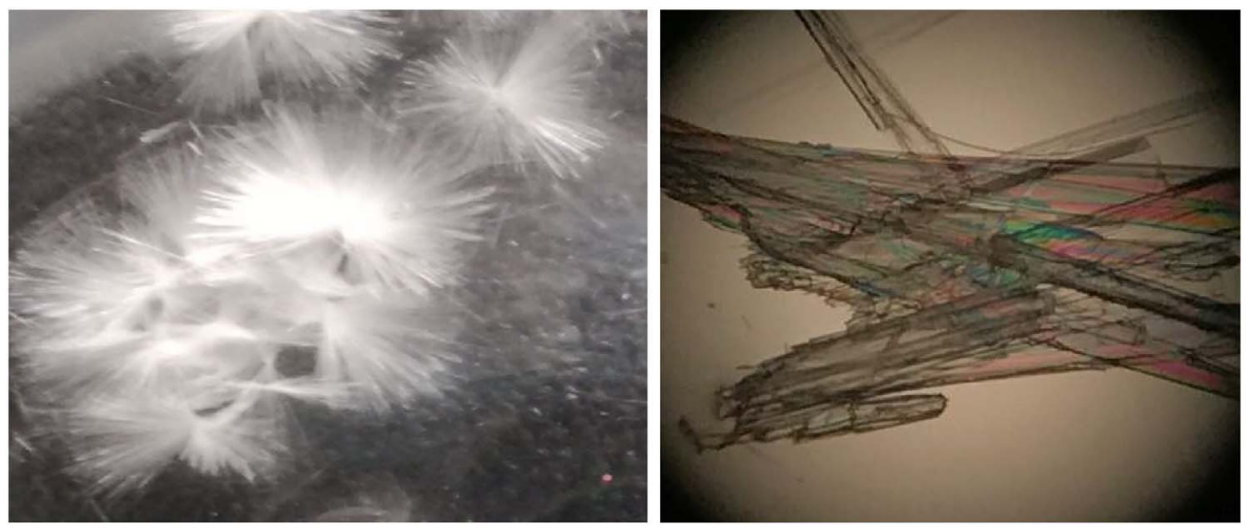

Fig. 5. Micrographs of DFA-PRO cocrystals.

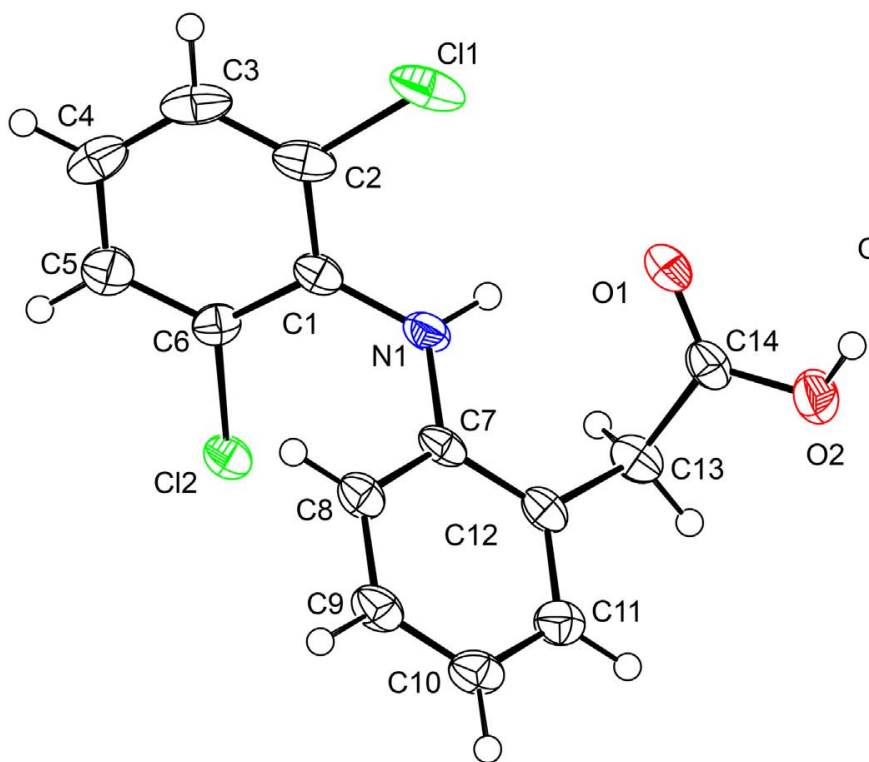

03

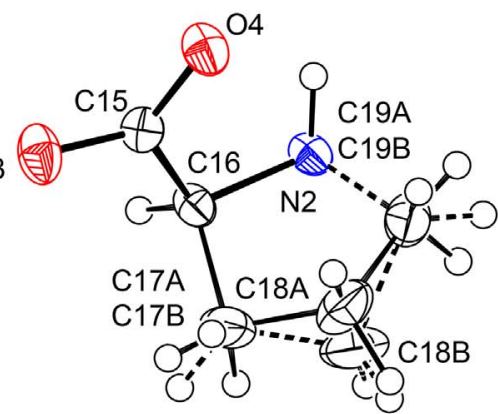

Fig. 6. Thermal ellipsoid drawing of the asymmetric unit in DFA-PRO drawn at 50\% probability level. The dashed line indicates the minor part of disordered PRO.

Table 1

Crystallographic data and refinement details of DFA-PRO.

\begin{tabular}{ll}
\hline Parameter & DFA-PRO \\
\hline Moiety formula & $\mathrm{C}_{14} \mathrm{H}_{11} \mathrm{Cl}_{2} \mathrm{NO}_{2}, \mathrm{C}_{5} \mathrm{H}_{9} \mathrm{NO}_{2}$ \\
Formula weight & 411.27 \\
Crystal system & Monoclinic \\
Space group & $P 2_{1}$ \\
$a(\AA)$ & $9.2875(7)$ \\
$b(\AA)$ & $5.2245(5)$ \\
$c(\AA)$ & $20.3755(17)$ \\
$\beta\left({ }^{\circ}\right)$ & $102.284(3)$ \\
Volume $\left(\AA^{3}\right)$ & $966.04(14)$ \\
$Z$ & 2 \\
T (K) & $93(2)$ \\
Measured ref. & 9569 \\
Independent ref. & $4183\left[R_{\text {(int) }}=0.0553\right]$ \\
Refined parameter & 265 \\
Goodness-of-fit on $F^{2}$ & 1.068 \\
Final $R$ indices $[I>2 \sigma(I)]$ & $R_{1}=0.0518$ \\
CCDC deposit number & 1584472 \\
\end{tabular}

each of DFA and PRO in the asymmetric unit (Fig. 6). The details of crystallographic data and refinement details of DFA-PRO shown in Table. 1. The pyrrolidine ring of the PRO molecule is disordered with the occupancy of major and minor parts determined at $0.85(2)$ and $0.15(2)$, respectively. Intramolecular $\mathrm{N} 1-\mathrm{H} 1 \cdots \mathrm{O} 1$ hydrogen bond
Table 2

Hydrogen bonds in DFA-PRO.

\begin{tabular}{lllll}
\hline$D-\mathrm{H} \cdots A$ & $D-\mathrm{H}(\AA)$ & $\mathrm{H} \cdots A(\AA)$ & $D \cdots A(\AA)$ & $D-\mathrm{H} \cdots A\left({ }^{\circ}\right)$ \\
\hline $\mathrm{N} 1-\mathrm{H} 1 \cdots \mathrm{Cl}^{\mathrm{c}}$ & $0.89(8)$ & $2.57(8)$ & $3.013(4)$ & $112(6)$ \\
$\mathrm{N}^{\mathrm{H}}-\mathrm{H} 1 \cdots \mathrm{O}^{\mathrm{c}}$ & $0.89(8)$ & $2.09(7)$ & $2.846(5)$ & $142(6)$ \\
$\mathrm{N}^{\mathrm{H}} \mathrm{H} 2 \mathrm{~A} \cdots 4^{\mathrm{c}}$ & $0.83(5)$ & $2.25(5)$ & $2.620(6)$ & $107(4)$ \\
$\mathrm{N} 2-\mathrm{H} 2 \mathrm{~B} \cdots \mathrm{O} 4^{\mathrm{a}}$ & $0.82(6)$ & $1.88(6)$ & $2.694(6)$ & $169(5)$ \\
$\mathrm{N} 2-\mathrm{H} 2 \mathrm{~A} \cdots \mathrm{O} 1$ & $0.83(5)$ & $2.17(5)$ & $2.819(5)$ & $136(4)$ \\
$\mathrm{O}^{\mathrm{O}}-\mathrm{H} 2 \cdots 3^{\mathrm{b}}$ & $0.87(3)$ & $1.68(3)$ & $2.543(5)$ & $169(6)$ \\
$\mathrm{C} 13-\mathrm{H} 13 \mathrm{~B} \cdots \mathrm{Cl}^{\mathrm{a}}$ & 0.99 & 2.78 & $3.646(5)$ & 145.9 \\
\hline
\end{tabular}

${ }^{\mathrm{a}} x, y-1, z$.

b $-x+1, y-1 / 2,-z$.

${ }^{\mathrm{c}}$ Intramolecular hydrogen bond.

stabilizes the conformation of the DFA molecule. A similar conformation is observed in all three polymorphs of DFA (Castellari and Ottani, 1997; Jaiboon et al., 2001). Crystal structure analysis revealed that DFA-PRO is a zwitterionic cocrystal. From the crystal structure, the $\mathrm{C}-\mathrm{O}$ distances between $\mathrm{C} 14-\mathrm{O} 1$ and $\mathrm{C} 14-\mathrm{O} 2$ in DFA are 1.225(6) $\AA$ and $1.311(5) \AA$, respectively. Since the difference in the $\mathrm{C}-\mathrm{O}$ distance is longer than $0.07 \AA(\Delta \mathrm{C}-\mathrm{O}>0.07 \AA)$, it confirms that the carboxylic acid remains in the DFA molecule. In the PRO molecule, $\mathrm{C}-\mathrm{O}$ distances of the carboxylate are comparable, which are 1.258(5) $\AA$ and 1.251(6) $\AA$ for $\mathrm{C} 15-\mathrm{O} 3$ and $\mathrm{C} 15-\mathrm{O} 4$, respectively, indicating its existence in zwitterionic form. 


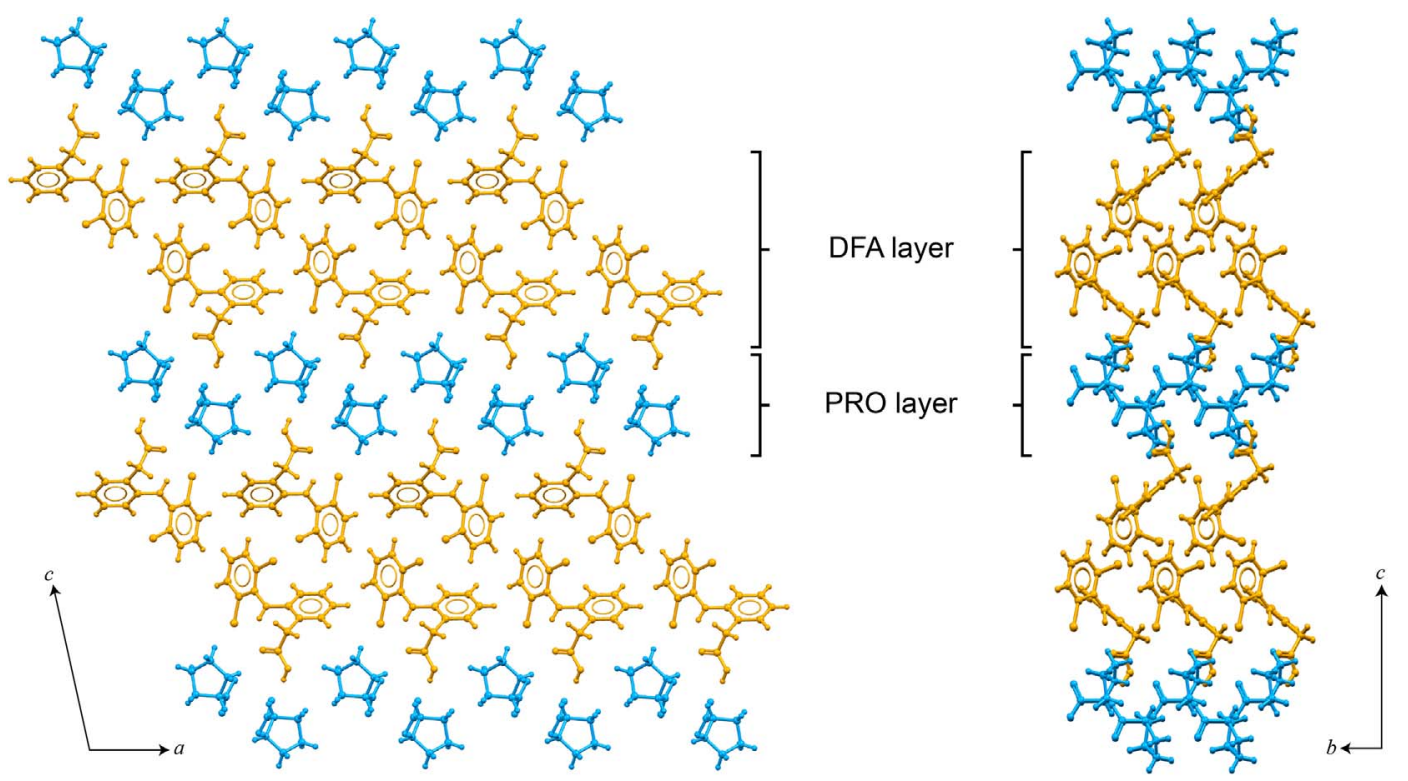

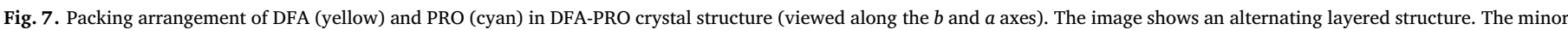
disordered part is omitted for clarity. (For interpretation of the references to color in this figure legend, the reader is referred to the web version of this article.)

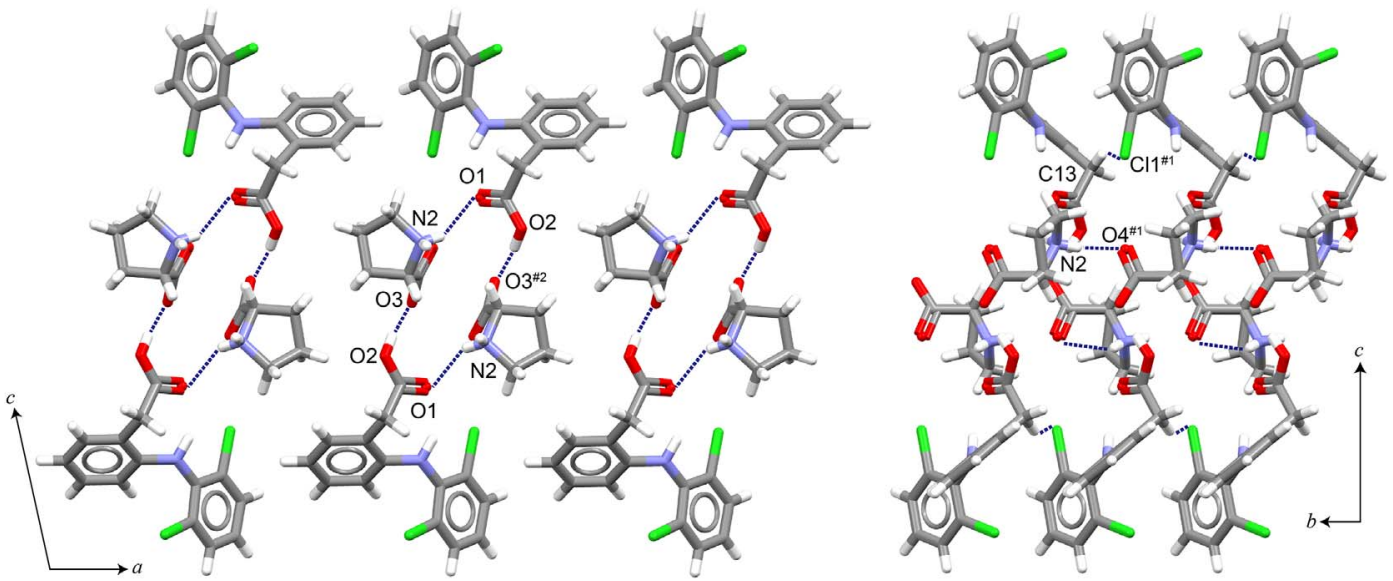

Fig. 8. Intermolecular interaction in DFA-PRO. The minor disordered parts are omitted for clarity. The symmetry codes are listed in Table 2.

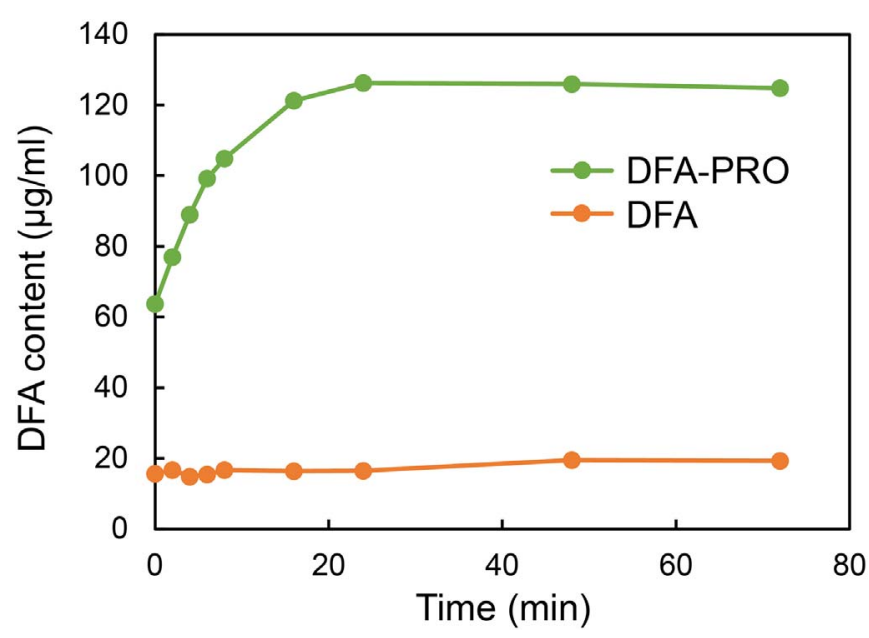

Fig. 9. Solubility profiles of DFA and DFA-PRO cocrystals as obtained by liquid assisted grinding.
Table 3

Powder solubility test results $(n=3)$.

\begin{tabular}{ll}
\hline Sample & Solubility $(\mu \mathrm{g} / \mathrm{mL})$ \\
\hline Pure diclofenac & $16.56 \pm 0.289$ \\
DFA-PRO obtained by liquid-assisted grinding & $125.91 \pm 0.322$ \\
\hline
\end{tabular}

The details of the hydrogen bonds are presented in Table 2. DFA and PRO formed an alternating layered structure (Fig. 7). The results show that, in the crystal structure, DFA molecules are connected to two adjacent PRO molecules via $\mathrm{O} 2-\mathrm{H} 2 \cdots \mathrm{O} 3$ and $\mathrm{N} 2-\mathrm{H} 2 \mathrm{~A} \cdots \mathrm{O} 1$ hydrogen bonds. Furthermore, DFA and PRO molecules are arranged as a chain structure along the crystallographic screw axis through C13-H13B $\cdots \mathrm{Cl} 1$ and N2-H2B $\cdots \mathrm{O} 4$ hydrogen bonds, respectively. The aforementioned instances of hydrogen bonding in DFA-PRO are described in Fig. 8. Details of the crystallographic data are listed in Table 1, whereas the hydrogen bonds that support the new co-arrangement are explained in Table 2.

Table 1 shows the crystal structure data as follows.

The hydrogen bond positions and its lengths are explained in Table 2 below. 

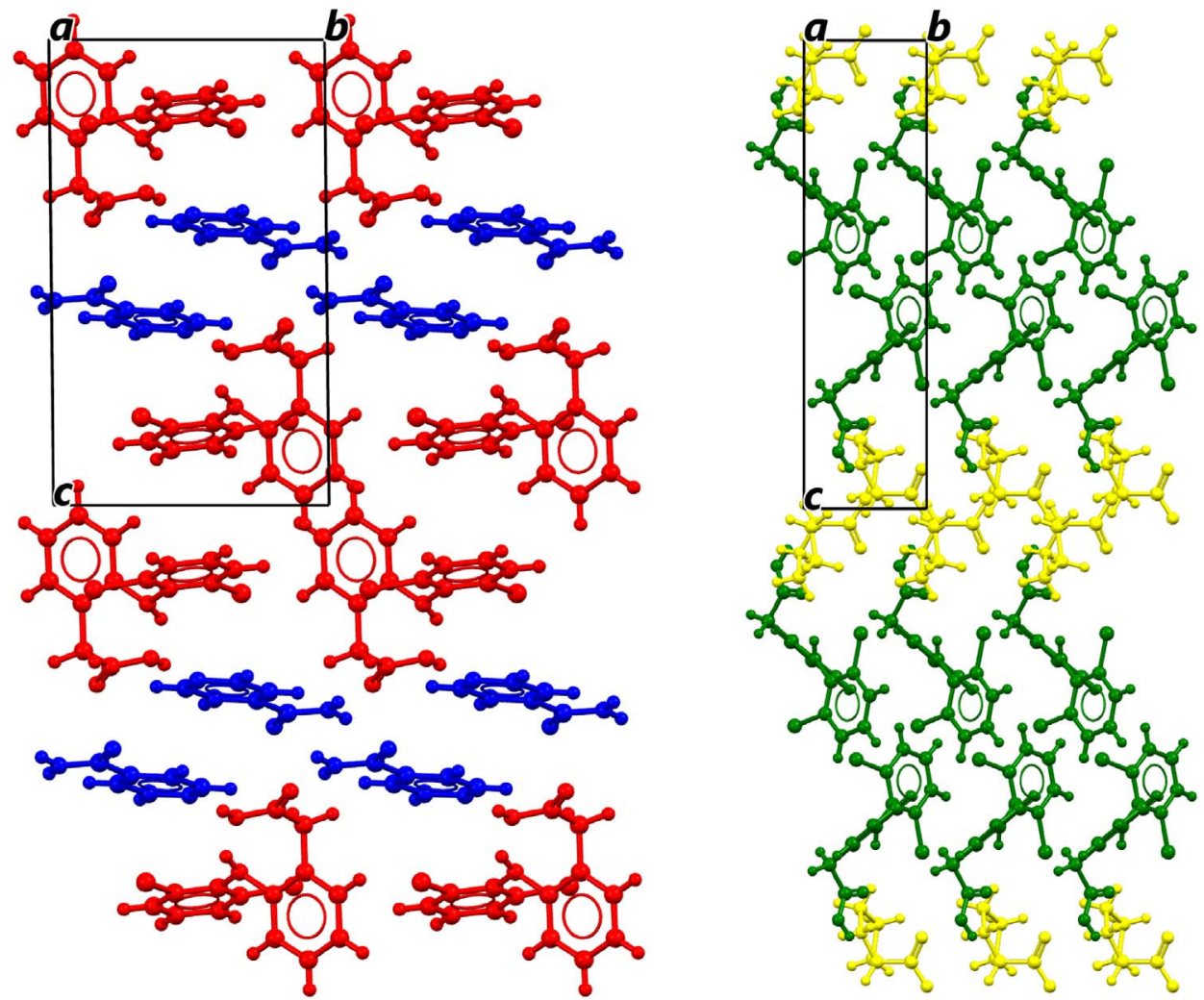

Fig. 10. Packing crystal structures of diclofenac-isonicotinamide (left) and DFA-PRO (right).

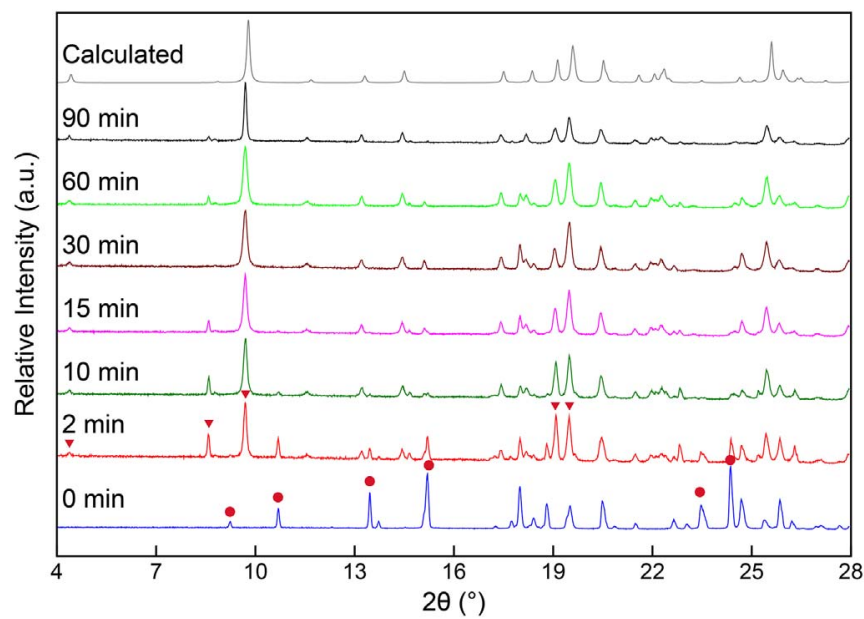

Fig. 11. Changes in the PXRD patterns of a physical mixture of DFA and PRO before and after co-grinding.

\subsubsection{Powder solubility in water}

The solubility profiles of diclofenac and its powder cocrystal are presented in Fig. 9 and Table 3. The solubility data indicates the concentration of DFA in the solution to each sample. The solubility of pure diclofenac and the cocrystal powder as shown in Table 3 are $16.56 \mu \mathrm{g} /$ $\mathrm{mL}$ and $125.91 \mu \mathrm{g} / \mathrm{mL}$, or equivalent with $0.056 \mathrm{mM}$ and $0.425 \mathrm{mM}$, respectively. From the results, the aqueous solubility of DFA-PRO is the 7.69-fold higher than that of the active compound.

The enhanced solubility of the DFA-PRO cocrystal can be explained according to its molecular arrangement and three-dimensional structure. Based on the data explained in Section 2.3.2, the layer structure of DFA-PRO is the main factor that contributed to the solubility enhancement. The DFA molecule is present on the channel sandwiched between two layers of PRO molecules. A similar molecular arrangement
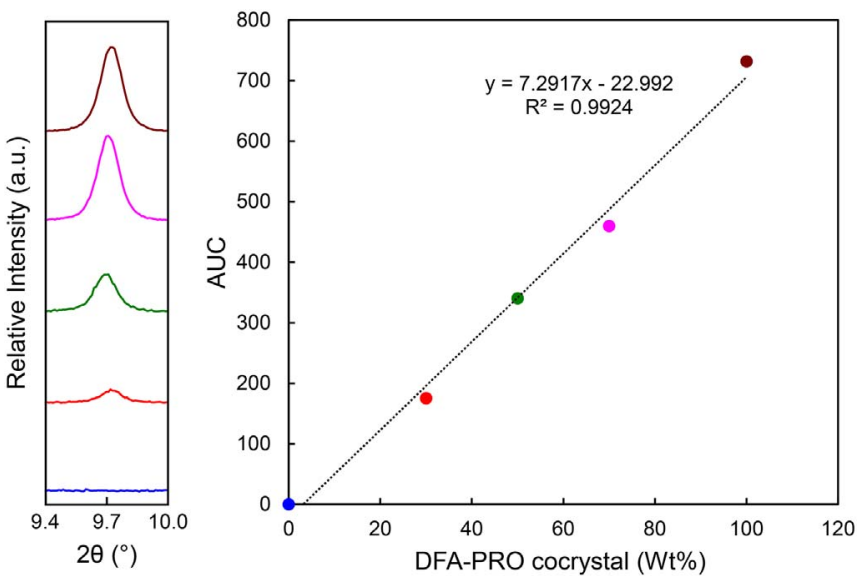

Fig. 12. PXRD patterns for a series of DFA-PRO standards (left) and the calibration curve obtained from them (right)

has been reported for epalrestat caffeine cocrystal (Putra et al., 2017) and naproxen-proline cocrystal (Tilborg et al., 2013). With such configuration, water molecules easily interact with the PRO molecules, resulting in a higher aqueous solubility (1300 g/L; Tilborg et al., 2014). Moreover, once the PRO molecules dissolved, the DFA-PRO interaction got broken, resulting in the exposure of the DFA molecules to the solvent. The enhanced solubility indicates that the DFA-PRO cocrystal is a promising candidate that can be developed into a solid dosage form. Moreover, L-proline is a nutritional substance, a GRAS compound, and a pharmacologically safe co-former (Wu et al., 2011); therefore, it is a suitable component of the cocrystal.

Aakeröy et al. (2011) recently described the use of pyridine or pyrimidine derivatives as co-formers in preparing diclofenac cocrystals; however, pyridine and pyrimidine derivatives are toxic (Liu et al., 1998; Sajewicz, 2007) to almost every vital organ in humans. 


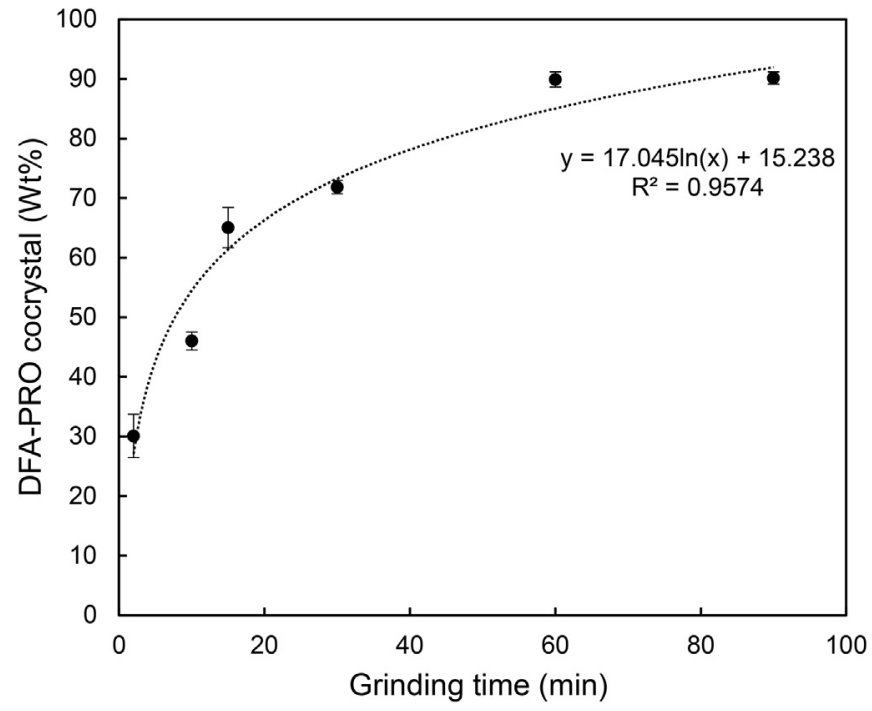

Fig. 13. Formation of DFA-PRO cocrystals during co-grinding at room temperature. Data are expressed as mean \pm standard deviation $(n=3)$.

Therefore, it is worth developing DFA-PRO cocrystals, which are a safer alternative and have enhanced bioavailability and pharmacological properties. Another recent report on diclofenac cocrystals is that by Báthori et al. (2011), which is on diclofenac-isonicotinamide cocrystals. Surov et al. (2014) also reported on diclofenac-theophylline cocrystal, and indicated that the solubility of the cocrystal was only 1.6-fold higher than that of pure diclofenac. The report on diclofenac-isonicotinamide cocrystal does not include data on the solubility of the cocrystal. According to the crystal structure, diclofenac-isonicotinamide cocrystal is also a layered-structure cocrystal. As shown in Fig. 10, the packing crystal structures of DFA-PRO and diclofenac-isonicotinamide are similar. Although the aqueous solubility of isonicotinamide $(191.7 \mathrm{mg} / \mathrm{mL})$ is higher than that of L-proline $(162 \mathrm{mg} / \mathrm{mL})$, it is difficult to suggest that the aqueous solubility of diclofenac-isonicotinamide is higher than that of DFA-PRO. Factors that influence the solubility of substances in water include lattice energy, molecular density, and co-former solubility (Good and Rodríguez-Hornedo, 2010).

\subsubsection{Kinetics of cocrystallization}

Cocrystals of DFA and PRO in equimolar amounts were formed by liquid-assisted grinding at room temperature to have a detailed insight into the kinetics of the co-grinding process during cocrystallization.

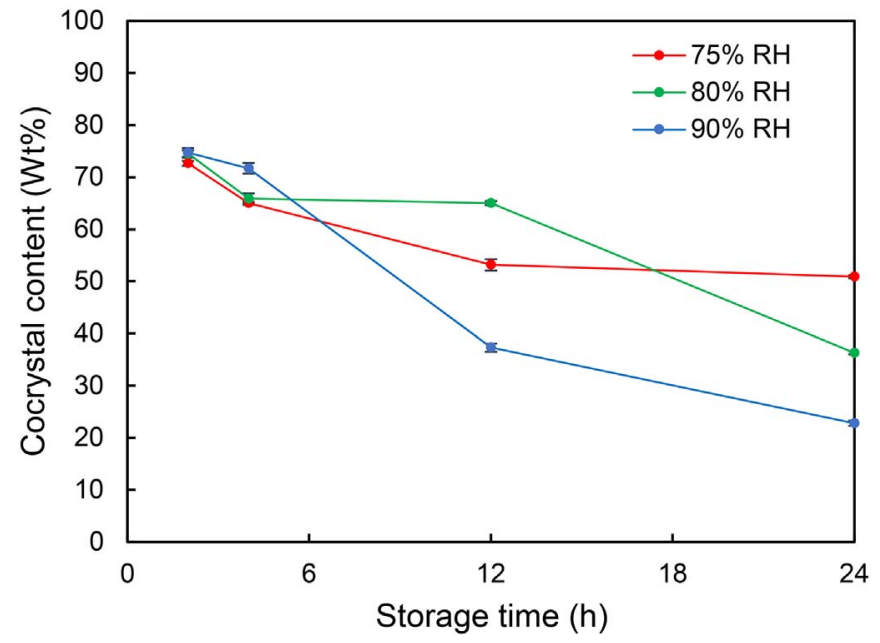

Fig. 15. Cocrystal content during storage at $30^{\circ} \mathrm{C}$ under various $\mathrm{RH}$ conditions $(75 \%$, $80 \%$, and $90 \% \mathrm{RH})$.

Changes in PXRD diffractogram during the process are seen in Fig. 11. Significant changes in the PXRD diffractogram occurred during the grinding process, which are indicative of the dynamic interaction between the molecules.

As seen in Fig. 11, there were several new reflection appearances (marked with closed triangles) in the diffractogram. However, some origin reflections disappeared (marked with dots). After $2 \mathrm{~min}$ of grinding, the characteristic reflections of DFA and PRO appeared predominantly at $2 \theta=10.60^{\circ}, 15.16^{\circ}$, and $24.35^{\circ}$ in the DFA diffractogram, and at $2 \theta=8.53^{\circ}$ and $19.15^{\circ}$ in the PRO diffractogram. The characteristic reflections of DFA-PRO cocrystal were also detected at $2 \theta=4.33^{\circ}$ and $9.64^{\circ}$. As grinding time was increased, the intensity of DFA-PRO increased, whereas that of both DFA and PRO decreased. Finally, after $90 \mathrm{~min}$ of grinding, the experimental diffractogram pattern was similar to the reference patterns obtained after evaporating the solvent from the single crystals. This indicated that the cocrystals had been completely formed.

Quantification of cocrystal amount was done according to the method described by Padrela et al. (2012). The initial step was selection of the characteristic diffraction peaks of DFA-PRO, which are at $2 \theta=9.71,14.47^{\circ}, 19.07^{\circ}$, and $19.54^{\circ}$. The best regression line equation for the quantification of DFA-PRO cocrystal was found to be $y=7.2917 x-22.992$. A high correlation coefficient of 0.9924 was obtained. A diffractogram of a standard series of DFA-PRO cocrystals
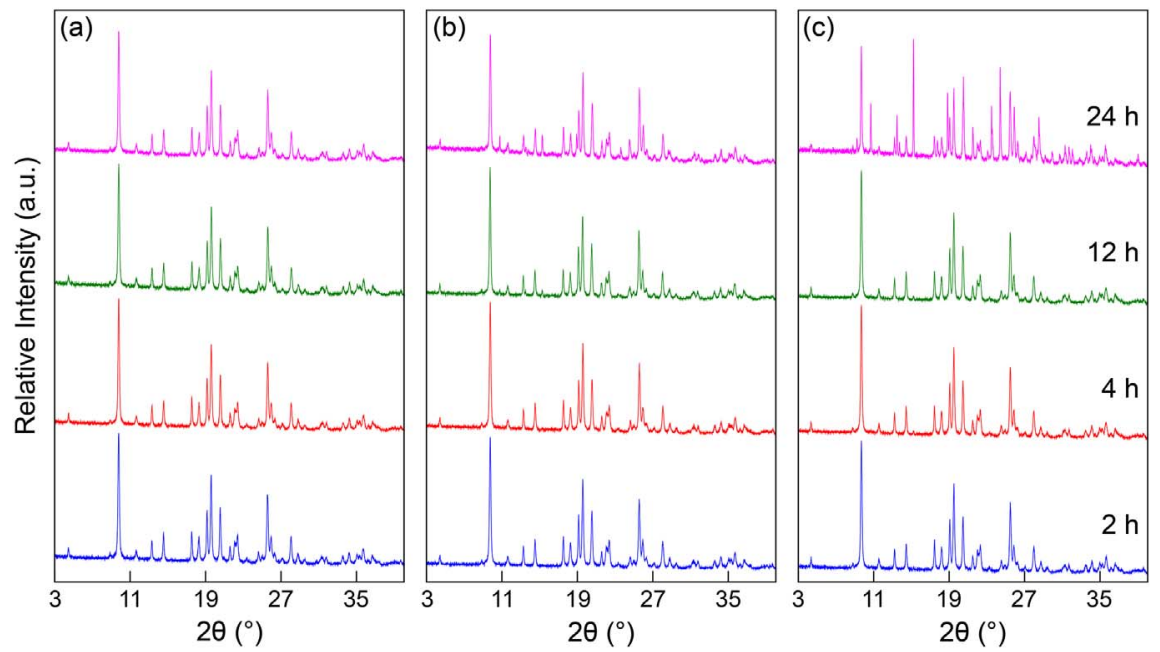

Fig. 14. PXRD patterns of DFA-PRO cocrystals after storage at $30{ }^{\circ} \mathrm{C}$ under various $\mathrm{RH}$ conditions ((a) $75 \%$, (b) $80 \%$, and (c) $90 \% \mathrm{RH}$ ). 
and the corresponding calibration curve are shown in Fig. 12. The calibration curve was used to determine the amount of the cocrystals (Wt $\%)$.

The amounts of DFA-PRO cocrystals formed at specific times during the grinding process are presented in Fig. 13. The amount of cocrystals formed increased gradually as grinding time was increased. After 60 and $90 \mathrm{~min}$ of grinding, the amounts obtained were $90.827 \pm 1.284$ and $90.878 \pm 1.011 \%$, respectively; however, the difference in the yields was not statistically significant. This indicates that DFA-PRO formation was complete after $60 \mathrm{~min}$ of grinding. The PXRD results obtained after $60 \mathrm{~min}$ of grinding corroborate these findings since similar diffractogram patterns were obtained for the cocrystals and single crystals (Fig. 11).

Cocrystal formation rate followed a logarithmic function as follows: $y=17.045 \ln x+15.238$. The correlation coefficient $\left(R^{2}\right)$ value was 0.9574 , which indicates first-order kinetics. The content of the cocrystals was not $90 \%$ after 90 min of grinding. This may be due to the use of the solvent to obtain the powder cocrystals by liquid-assisted grinding. Furthermore, crystal defects or amorphous regions were detected in the crystals due to the addition of solvent during the liquidassisted grinding process. Similar results were obtained during the quantification of indomethacin-saccharin cocrystals produced by liquid-assisted grinding (Padrela et al., 2012).

\subsubsection{Stability study}

In order to ensure effective and widespread use of cocrystals, a greater understanding of factors such as polymorphism and physical stability is necessary (Eddleston et al., 2014). The stabilities of active pharmaceutical ingredients (APIs) and dosage forms during distribution and storage depend on two major factors: their chemical and physical properties, and environmental factors. Pharmaceutical cocrystals exhibit specific stability characteristics. For instance, certain APIs and coformers tend to dissociate during storage, particularly under high humidity and temperature conditions. Additionally, removal or alteration of a co-former in a cocrystal destroys the crystal arrangement and changes the parent API to its original form (Hsu et al., 2012). Trask et al. (2006) studied the stability of cocrystals of dicarboxylic acid, caffeine, and theophylline at high humidity and found that the cocrystals dissociated within seven days at $98 \% \mathrm{RH}$. This resulted in crystallization of the acid and caffeine/theophylline hydrate as separate phases. Due to the importance of stability in the development of new cocrystals, the stability of the cocrystals was investigated at $30{ }^{\circ} \mathrm{C}$ under various humidity conditions $(75 \%, 80 \%$, and $90 \% \mathrm{RH})$.

Fig. 13 shows diffractogram of the DFA-PRO cocrystals at $30^{\circ} \mathrm{C}$ during storage at $75 \%, 80 \%$, or $90 \% \mathrm{RH}$ for $24 \mathrm{~h}$. The results indicate that the cocrystals were relatively stable at $75 \% \mathrm{RH}$, as no changes in pattern or intensity were observed (Fig. 14(a)). However, a propensity for decomposition occurred at $80 \% \mathrm{RH}$ and further increased at $90 \%$ $\mathrm{RH}$. After $24 \mathrm{~h}$ of storage at $80 \% \mathrm{RH}$, the cocrystals partially dissociated, which was evidenced by a low trace intensities at $2 \theta=10.60^{\circ}$, $15.16^{\circ}, 18.78^{\circ}$, and $23.50^{\circ}$ in the diffractogram of DFA (Fig. 14(b)). Moreover, diffraction of PRO was detected at $2 \theta=19.4^{\circ}, 23.45^{\circ}$, and $25.46^{\circ}$ at high intensities. A similar phenomenon was observed after $12 \mathrm{~h}$ of storage at $90 \% \mathrm{RH}$; however, the intensity was higher than that at $80 \% \mathrm{RH}$. The findings show that the stability of DFA-PRO cocrystals is influenced by humidity. However, no solid-state alteration of the parent compound (i.e. polymorphism) was observed during the storage period.

In order to further understand the dissociation of DFA-PRO due to humidity, the amount of the cocrystals was measured after storage under the various conditions. As shown in Fig. 15, the amount of the cocrystals decreased gradually during storage, and the rate of decrease was related to humidity. The dissociation reaction order followed a first-order reaction at all conditions. This indicates that the dissociation of DFA-PRO cocrystals is proportional to cocrystal concentration and increases in a logarithmic order. The following logarithmic equations were obtained at the various RH conditions.

$\mathrm{y}=-9.08 \ln \mathrm{x}+78.009 ; \mathrm{R} 2=0.9705(70 \% \mathrm{RH})$

$\mathrm{y}=-13 \ln \mathrm{x}+85.608 ; \mathrm{R} 2=0.9039(80 \% \mathrm{RH})$

$\mathrm{y}=-22.6 \ln \mathrm{x}+95.364 ; \mathrm{R} 2=0.9555(90 \% \mathrm{RH})$

The phenomenon observed in this experiment can be explained by considering the humidity-induced dissociation of caffeine-theophylline cocrystals reported by Trask et al. (2005). In an environment of high humidity, the components of the DFA-PRO cocrystals tend to form hydrated phases. If the free energy of the co-former or API hydrated phase is lower than that of the cocrystal, separation of the two molecules will be favored. Therefore, it could be presumed that dissociation of DFA-PRO cocrystals under high humidity conditions is caused by the presence of water, which is absorbed by L-proline to form a hydrate. However, the exact mechanism underlying the dissociation of DFA-PRO cocrystals in a humid environment is not completely explained by the findings of the current study. Therefore, future studies on this should be considered.

\subsection{Conclusion}

A new zwitterionic cocrystal of diclofenac and L-proline was successfully formed and fully characterized in this study. The results showed that the DFA-PRO cocrystals are needle-shaped with a layered crystal structure that is advantageous in improving their aqueous solubility. The cocrystals were found to be 7 times more soluble in water than DFA. Furthermore, formation of the cocrystals was found to follow first-order reaction kinetics. In the physical stability test, the DFA-PRO cocrystals were found to be stable at $30^{\circ} \mathrm{C} / 75 \% \mathrm{RH}$; however, they tended to dissociate into DFA and L-proline monohydrate at higher humidity conditions ( $80 \%$ and $90 \% \mathrm{RH})$. The comprehensive characterization conducted in this study can serve as a guide when determining the physicochemical properties of zwitterionic cocrystals, particularly when solubility and stability characteristics are desired.

\section{Acknowledgement}

This research was supported by World Class University Program: International Research Collaboration between LPPM-ITB (Jawa Barat, Indonesia) with Department of Chemistry and Material Science, Tokyo Institute of Technology, Japan (grant number: 007/WCU-ITB/LL/III/ 2017).

\section{References}

Aakeröy, C.B., Grommet, A.B., Desper, J., 2011. Co-crystal screening of diclofenac. Pharmaceutics 3, 601-614. http://dx.doi.org/10.3390/pharmaceutics3030601.

Barbato, F., Cappello, B., La Rotonda, M.I., Miro, A., Quaglia, F., 2003. Diclofenac ßcyclodextrin binary systems: a study in solution and in the solid state. J. Incl. Phenom. Macrocycl. Chem. 46, 179-185. http://dx.doi.org/10.1023/ A:1026303516401.

Báthori, N.B., Lemmerer, A., Venter, G.A., Bourne, S.A., Caira, M.R., 2011 Pharmaceutical co-crystals with isonicotinamide-vitamin B3, clofibric acid, and diclofenac-and two isonicotinamide hydrates. Cryst. Growth Des. 11, 75-87. http:// dx.doi.org/10.1021/cg100670k.

Bhandaru, J.S., Malothu, N., Akkinepally, R.R., 2015. Characterization and solubility studies of pharmaceutical cocrystals of eprosartan mesylate. Cryst. Growth Des. 15, 1173-1179. http://dx.doi.org/10.1021/cg501532k.

Bruno, I.J., Cole, J.C., Edgington, P.R., Kessler, M., Macrae, C.F., McCabe, P., Pearson, J., Taylor, R., 2002. New software for searching the Cambridge Structural Database and visualizing crystal structures. Acta Crystallogr. B 58, 389-397. http://dx.doi.org/10. $1107 /$ S0108768102003324.

Castellari, C., Ottani, S., 1997. Two monoclinic forms of diclofenac acid. Acta Crystallogr. C Struct. Chem. 53, 794-797. http://dx.doi.org/10.1107/S0108270197002126.

Duggirala, N.K., Perry, M.L., Almarsson, Ö., Zaworotko, M.J., 2016. Pharmaceutical cocrystals: along the path to improved medicines. Chem. Commun. 52, 640-655. http://dx.doi.org/10.1039/C5CC08216A.

Eddleston, M.D., Thakuria, R., Aldous, B.J., Jones, W., 2014. An investigation of the causes of cocrystal dissociation at high humidity. J. Pharm. Sci. 103, 2859-2864. http://dx.doi.org/10.1002/jps.23865. 
Fini, A., Cavallari, C., Ospitali, F., 2010. Diclofenac salts. V. Examples of polymorphism among diclofenac salts with alkyl-hydroxy amines studied by DSC and HSM. Pharmaceutics 2, 136-158. http://dx.doi.org/10.3390/pharmaceutics2020136.

Fini, A., Bassini, G., Monastero, A., Cavallari, C., 2012. Diclofenac salts, VIII. Effect of the counterions on the permeation through porcine membrane from aqueous saturated solutions. Pharmaceutics 4, 413-429. http://dx.doi.org/10.3390/ pharmaceutics 4030413.

Good, D.J., Rodríguez-Hornedo, N., 2010. Cocrystal eutetic constants and prediction of solubility behavior. Cryst. Growth Des. 10, 1028-1032. http://dx.doi.org/10.1021/ cg901232h.

He, H., Huang, Y., Zhang, Q., Wang, J.R., Mei, X., 2016. Zwitterionic cocrystals of flavonoids and proline: solid-state characterization, pharmaceutical properties, and pharmacokinetic performance. Cryst. Growth Des. 16, 2348-2356. http://dx.doi.org/ 10.1021/acs.cgd.6b00142.

Higashi, T., 1995. ABSCOR. Rigaku Corporation, Tokyo, Japan.

Hsu, P.C., Lin, H.L., Wang, S.L., Lin, S.Y., 2012. Solid-state thermal behavior and stability studies of theophylline-citric acid cocrystals prepared by neat cogrinding or thermal treatment. J. Solid State Chem. 192, 238-245. http://dx.doi.org/10.1016/j.jssc. 2012.04.010.

Jaiboon, N., Yos-In, K., Ruangchaithaweesuk, S., Chaichit, N., Thutivoranath, R., Siritaedmukul, K., Hannongbua, S., 2001. New orthorhombic form of 2-[(2,6-dichlorophenyl)amino]benzeneacetic acid (diclofenac acid). Anal. Sci. 17, 1465-1466. http://dx.doi.org/10.2116/analsci.17.1465.

Liu, S.M., Wu, C.H., Huang, H.J., 1998. Toxicity and anaerobic biodegradability of pyridine and its derivatives under sulfidogenic conditions. Chemosphere 36, 2345-2357. http://dx.doi.org/10.1016/S0045-6535(97)10203-X.

Liu, M., Hong, C., Yao, Y., Shen, H., Ji, G., Li, G., Xie, Y., 2016. Development of a pharmaceutical cocrystal with solution crystallization technology: preparation, characterization, and evaluation of myricetin-proline cocrystals. Eur. J. Pharm. Biopharm. 107, 151-159. http://dx.doi.org/10.1016/j.ejpb.2016.07.008.

Macrae, C.F., Edgington, P.R., McCabe, P., Pidcock, E., Shields, G.P., Taylor, R., Towler, M., van de Streek, J., 2006. Mercury: visualization and analysis of crystal structures. J. Appl. Crystallogr. 39, 453-457. http://dx.doi.org/10.1107/S002188980600731X.

Macrae, C.F., Bruno, I.J., Chisholm, J.A., Edgington, P.R., McCabe, P., Pidcock, E. Rodriguez-Monge, L., Taylor, R., van de Streek, J., Wood, P.A., 2008. Mercury CSD 2.0 - new features for the visualization and investigation of crystal structures. J. Appl. Crystallogr. 41, 466-470. http://dx.doi.org/10.1107/S0021889807067908.

Othman, M.F., Jamburi, N., Anuar, N., Abd Rahim, S., Rohalim, N.H., 2016. Ibuprofenamino acids co-crystal screening via co-grinding methods. MATEC Web Conf. 69 , 03002. http://dx.doi.org/10.1051/matecconf/20166903002.

Padrela, L., de Azevedo, E.G., Velaga, S.P., 2012. Powder X-ray diffraction method for the quantification of cocrystals in the crystallization mixture. Drug Dev. Ind. Pharm. 38, 923-929. http://dx.doi.org/10.3109/03639045.2011.633263.

Putra, O.D., Umeda, D., Nugraha, Y.P., Furuishi, T., Nagase, H., Fukuzawa, K., Uekusa, H.,
Yonemochi, E., 2017. Solubility improvement of epalrestat by layered structure formation via cocrystallization. CrystEngComm 19, 2614-2622. http://dx.doi.org/ 10.1039/C7CE00284J.

Qiu, J.B., Li, G., Sheng, Y., Zhu, M.R., 2015. Quantification of febuxostat polymorphs using powder X-ray diffraction technique. J. Pharm. Biomed. Anal. 107, 298-303. http://dx.doi.org/10.1016/j.jpba.2015.01.005.

Sajewicz, W., 2007. Toxicity of pyrimidine derivatives under oxidative stress conditions: chemiluminescence-based assays in systems containing erythrocytes, mitochondria or blood plasma. Pharmacol. Rep. 59, 206-215.

Sheldrick, G.M., 2015a. SHELXT - integrated space-group and crystal-structure determination. Acta Crystallogr., Sect. A: Found. Adv. 71, 3-8. http://dx.doi.org/10.1107/ S2053273314026370.

Sheldrick, G.M., 2015b. Crystal structure refinement with SHELXL. Acta Crystallogr. C Struct. Chem. 71, 3-8. http://dx.doi.org/10.1107/S2053229614024218.

Silva, J.L.A.F., Santos, P.P., André, V., Galego, F., 2016. New cocrystals of flurbiprofen and proline: structural effect of enantiomorphism. Acta Crystallogr., Sect. A: Found. Adv. 72, s356. http://dx.doi.org/10.1107/S2053273316094730.

Surov, A.O., Voronin, A.P., Manin, A.N., Manin, N.G., Kuzmina, L.G., Churakov, A.V., Perlovich, G.L., 2014. Pharmaceutical cocrystals of diflunisal and diclofenac with theophylline. Mol. Pharm. 11, 3707-3715. http://dx.doi.org/10.1021/mp5004652.

Tilborg, A., Springuel, G., Norberg, B., Wouters, J., Leyssens, T., 2013. On the influence of using a zwitterionic coformer for cocrystallization: structural focus on naproxen-proline cocrystals. CrystEngComm 15, 3341-3350. http://dx.doi.org/10.1039/ C3CE40084K.

Tilborg, A., Norberg, B., Wouters, J., 2014. Pharmaceutical salts and cocrystals involving amino acids: a brief structural overview of the state-of-art. Eur. J. Med. Chem. 74, 411-426. http://dx.doi.org/10.1016/j.ejmech.2013.11.045.

Tiwari, M., Chawla, G., Bansal, A.K., 2007. Quantification of olanzapine polymorphs using powder X-ray diffraction technique. J. Pharm. Biomed. Anal. 43, 865-872. http://dx.doi.org/10.1016/j.jpba.2006.08.030.

Trask, A.V., Samuel Motherwell, W.D., Jones, W., 2005. Pharmaceutical cocrystallization: engineering a remedy for caffeine hydration. Cryst. Growth Des. 5, 1013-1021. http://dx.doi.org/10.1021/cg0496540.

Trask, A.V., Sam Motherwell, W.D., Jones, W., 2006. Physical stability enhancement of theophylline via cocrystallization. Int. J. Pharm. 320, 114-123. http://dx.doi.org/10. 1016/j.ijpharm.2006.04.018.

Williams, B.S., Buvanendran, A., 2011. Nonopioid analgesics: NSAIDs, COX-2 inhibitors, and acetaminophen. In: Benzon, H., Raja, S.N., Fishman, S.M., Liu, S.S., Cohen, S.P. (Eds.), Essentials of Pain Medicine. Elsevier, Philadelphia, pp. 130-139. http://dx. doi.org/10.1016/B978-1-4377-2242-0.00026-2.

Wu, G., Bazer, F.W., Burghardt, R.C., Johnson, G.A., Kim, S.W., Knabe, D.A., Li, P., Li, X., McKnight, J.R., Satterfield, M.C., Spencer, T.E., 2011. Proline and hydroxyproline metabolism: implications for animal and human nutrition. Amino Acids 40, 1053-1063. http://dx.doi.org/10.1007/s00726-010-0715-z. 